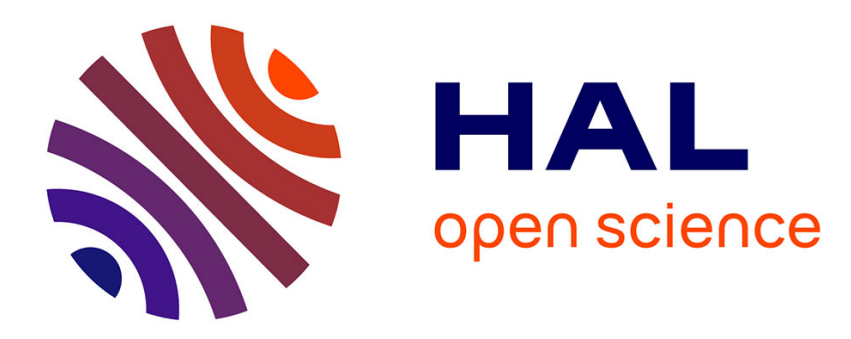

\title{
Automatisation du métro et qualité de service
} Robin Foot

\section{- To cite this version:}

Robin Foot. Automatisation du métro et qualité de service. Automatisation et transport, 1988, Paris, France. pp.36-59. halshs-00437523

\section{HAL Id: halshs-00437523 https://shs.hal.science/halshs-00437523}

Submitted on 30 Nov 2009

HAL is a multi-disciplinary open access archive for the deposit and dissemination of scientific research documents, whether they are published or not. The documents may come from teaching and research institutions in France or abroad, or from public or private research centers.
L'archive ouverte pluridisciplinaire HAL, est destinée au dépôt et à la diffusion de documents scientifiques de niveau recherche, publiés ou non, émanant des établissements d'enseignement et de recherche français ou étrangers, des laboratoires publics ou privés. 
(1988) Cahier du GDR Réseaux, I I: 36-59

\section{AUTOMATISATION DU METRO et QUALITE DE SERVICE}

Robin FOOT

Juin 1988

Le 13 décembre 1985, le SYTRAL (Syndicat Mixte des Transports pour le Rhône et l'Agglomération Lyonnaise), propriétaire du réseau, prend la décision d'automatiser intégralement la conduite et la surveillance des rames de métro sur la ligne $\mathrm{D}$ alors que les travaux de construction de celle-ci sont déjà entamés depuis 1983. Le réseau de transport en commun de l'agglomération lyonnaise, par cette décision, crée l'événement dans le monde du transport urbain par métro lourd et ce pour au moins deux raisons. 
La première réside dans l'option stratégique prise de substituer intégralement, sur un métro lourd à grand gabarit, des automates aux conducteurs. Jusque-là, seule l'automatisation des réseaux de type métro léger (Vancouver, Kobe, Osaka et Lille) ont permis la suppression des conducteurs. Sur les métros à grand gabarit, les processus d'automatisation conduisent, par le biais du pilotage automatique, à transformer radicalement le rôle des conducteurs vers une "action" de surveillance tant en direction des automates que du public sans toutefois autoriser leur suppression. Là, dans le projet lyonnais, la conception retenue du système métro exclut toute idée de mise en place d'une forme permanente "d'agent d'accompagnement des rames" dans l'organisation de la production de transport. Le pari retenu consiste à estimer que les avancées techniques sont arrivées à un degré de maturité tel qu'il est possible de réaliser un métro lourd complètement automatique assurant au moins un même niveau de sécurité et de qualité de service pour les voyageurs, qu'un métro de type classiques avec conducteurs et de à des conditions économiques favorables.

La seconde raison du caractère innovateur du projet d'automatisation tient à la date de prise de décision. Quand l'accord est donné, fin 1985, non seulement les travaux de réservation, de déviation de réseaux de service public mais aussi une partie importante des travaux de génie civil sont déjà réalisés. En effet, toutes les études menées par la SEMALY (Société d'Economie Mixte du Métropolitain de l'Agglomération Lyonnaise) pour la construction de la ligne D ont été exécutées dans la perspective d'une compatibilité globale des trois lignes sur pneumatique du métro lyonnais, en termes de techniques ferroviaires, afin de permettre une ouverture progressive de la 38 ligne D. A l'origine du projet ligne D, l'ouverture est prévue en deux étapes. En 1987, la première tranche de la ligne D, Saint-Jean Vieux Lyon/Gare de Vénissieux (9,2km et 12 stations) devait être ouverte au public. Les seules évolutions portent sur le matériel roulant, avec de la microinformatique embarquée, et une refonte globale du Poste de Commande Centralisée. Le constat au bout de cinq ans d'exploitation d'une mauvaise conception d'ensemble et d'une obsolescence technique rapide ont imposé cette dernière modification. La décision de 1985 d'automatiser la circulation des rames, en rupture avec ce choix de cohérence du réseau métro, équivaut, compte tenu de l'état d'avancement de la ligne, à une automatisation d'un réseau existant. Ce décalage dans la prise de décision se "matérialise" de manière 
spectaculaire par l'absence d'un système de type "portes palières" pour assurer la clôture de la "frontière" entre le quai et la voie.

Ces deux aspects du projet MAGGALY (Métro Automatique à Grand Gabarit de l'Agglomération Lyonnaise) fondent le caractère innovant du projet. L'enjeu de cette expérimentation dépasse même s'il s'inscrit complètement dans l'agglomération lyonnaise, les intérêts locaux des acteurs du transport en commun de Lyon. De la réussite tant en termes économiques qu'en termes de qualité de service, de MAGGALY dépend l'ouverture d'un marché potentiel considérable, celui de la modernisation des réseaux métro existants pour les constructeurs français.

Dans cette communication, nous centrerons notre questionnement sur deux points:

- les processus de constitution d'une maîtrise technique et technologique, la lente et longue émergence des principes de constitution d'un réseau de transport :

- à l'intérieur de ce processus comment sont désignés, au travers de la technique, les conducteurs, comment la formalisation technique nomme le rôle d'un groupe de salariés dans le procès de production d'ensemble.

Nous essaierons de comprendre l'articulation entre ces deux points dans la technique car le système technique traduit non seulement une représentation du groupe des conducteurs mais aussi et peut-être surtout la vision qu'on les concepteurs de système de la production de transport dans son ensemble. La représentation des logiques productives des concepteurs et dirigeants dessinent une image sociale des conducteurs, projetée en reflets par la technique.

\section{DES EXPERIENCES LOCALES REVELATRICES DES RAPPORTS ENTRE TECHNIQUE ET TRAVAIL.}

Il nous paraît important, compte tenu de la primauté et de l'importance du réseau métro de la RATP, de remonter quelque peu dans le temps afin de percevoir la

R. Foot (1988) Automatisation du métro et qualité de service Cahier du GDR Réseaux, 11: 36-59 
transformation radicale qui s'est opérée dans la représentation des conducteurs dans le procès de production métro.

Les expérimentations des années 50 ont porté sur des aspects particuliers et différents de l'organisation du travail et de la production. Du fait de leur caractère expérimental, on peut voir se matérialiser, autour d'un niveau technologique donné, le champ des possibles en matière de conception de la production et de réorganisation du travail dans les processus d'innovation. Le choix final, l'option du métro sur pneumatique, retenu comme axe de développement du métro à la fin de cette période, nous permettra de lire à la fois en positif, dans l'image du conducteur, et en négatif, la représentation du système par les concepteurs, la logique qui s'affirme.

On s'arrêtera sur quatre modernisations qui se déroulent sur deux lignes du réseau:

- le matériel articulé et la centralisation des communications sur la ligne 13 (ligne en fourche de Saint-Lazare à Carrefour Pleyel ou Porte de Clichy);

- le matériel pneumatique et le Pilotage Automatique sur la voie navette Porte des Lilas - Pré-Saint-Gervais.

\subsection{Le matériel articulé.}

La livraison du matériel articulé à la fin 1951, rompt avec une période de plus de quinze ans où aucun nouveau matériel n'a été mis en service. Avec l'introduction du courant basse tension, une loge plus grande, l'équipe de train se retrouve à travailler ensemble dans la loge $\mathrm{e}^{1}$.

L’attelage et le dételage du matériel articulé fondait sa spécificité :

1 Le matériel articulé, mis à l'étude en 1938, visait à permettre une exploitation par rames déformables. A la différence des rames Sprague-Thomson, composées de voitures d'environ 14 mètres à deux bogies, le matériel articulé se compose d'éléments d'environ 36 mètres de long.

R. Foot (1988) Automatisation du métro et qualité de service Cahier du GDR Réseaux, 11: 36-59 
"Peu avant la guerre, on avait mis en doute le bien-fondé de l'exploitation de certaines lignes par rames indéformables et on avait envisagé la construction d'un matériel dont les rames pourraient être sectionnées facilement en deux parties égaies, permettant, selon l'affluence, de former des trains de longueurs variables".

La possibilité d'adaptation au trafic voyageur qu'offrait ce matériel n'a pas pu être pratiquement utilisée car le profil des lignes et la configuration des terminus ne permettent pas une exploitation aisée de ce matériel articulé.

\subsection{Un système centralisé de communication.}

Sur cette ligne fut également expérimenté un réseau centralisé de communication d'information et de commandement. Avec trois terminus et une fourche "déséquilibrée", les problèmes de communication et de régulation, sur cette ligne, se posaient avec encore plus d'acuité que sur les autres. Un poste de "régulation centrale" fut installé, en 1953, à Saint-Lazare:

“À partir de postes téléphoniques spéciaux placés au bout des quais, les conducteurs devaient, à l'arrêt des trains, signaler leur retard au chef de régulation du terminus Saint-Lazare, lequel pouvait retenir des trains en station en commandant directement des feux de Départ Sur Ordre (DSO) placés à la sortie de ces stations"’.

Cette centralisation des communications allait de pair avec les premiers chaînages logiques des mesures de régulation. Le décrochement d'un combiné téléphonique en tête de quai par un membre de l'équipe de train, déclenchait automatiquement les voyants lumineux du Départ Sur Ordre de la station amont. Cette automaticité locale d'une mesure de régulation partait du principe que la

2 Le métro des origines à nos jours, RATP, 1984, p. 17. C'est cette solution qui est adoptée pour le RER

3 "Les commandes centralisées du métro urbain de Paris", octobre 1973, p. 1. Extrait de la Revue générale des chemins de fer. mai 1973.

R. Foot (1988) Automatisation du métro et qualité de service Cahier du GDR Réseaux, 11: 36-59 
demande d'une communication signifiait forcément un sur-stationnement et que, préventivement, il valait mieux retenir la rame suivante avant qu'elle ne s'engage sous tunnel.

Avec ce poste de régulation, une nouvelle dénomination apparaît: le chef régulateur. Doté d'un tableau de contrôle optique, il devient le personnage clé de la ligne. C'est en quelque sorte, sous une forme locale, la préfiguration du poste de contrôle centralisée actuelle.

Cet aspect de la modernisation de la ligne 13 révèle clairement que sans modification du matériel roulant, avec "simplement" une réorganisation du système téléphonique et la mise en place d'une télécommande de départ sur ordre, les points les plus évidents de "dérégulation" pouvaient être résolus dès cette époque.

Mais cette restructuration du système de communication entraîne une transformation sensible de la division du travail dans le collectif de ligne. Le regroupement dans un même espace, la loge, du chef de train (Mouvement) et du conducteur (Traction) et la centralisation des communications, rende le terminus névralgique pour la Maîtrise

Mouvement et Traction: cela restructure de fait l'organisation de la division du travail en ligne non plus tant par rapport aux filières que par rapport aux fonctions et espaces de travail.

La non réalisation, dès cette époque, d'une centralisation des communications, point pivot d'une "modernisation" de l'organisation d'ensemble du métro, paraît avoir en partie comme origine, le refus de restructurer les collectifs de travail, de toucher aux divisions en filières de l'organisation sociale du métro. La généralisation d'un tel système de communication aurait matérialisé un rapport de subordination de la Traction vis-à-vis du Mouvement.

\subsection{Le pilotage automatique.}

À l'écart du réseau, sur la petite voie navette longue de 750 mètres, entre Porte des Lilas et Pré Saint-Gervais, des ingénieurs de la RATP et de la Compagnie de 
Signaux et d'Entreprises Electriques (C.S.E.E.) saisiront l'occasion de la mise en service des "matériels pneumatiques", en 1952, pour expérimenter et mettre au point un système de Pilotage Automatique.

Le Pilotage Automatique, basé sur un "dialogue" entre un système embarqué et un système programmé au sol, permet par actionnement du freinage ou de la traction de contrôler automatiquement les vitesses et les arrêts. Cette expérience dure quatre ans puis est interrompue sans que l'on en connaisse précisément les raisons. Les explications officielles sont en effet très lacunaires :

"Bien que cette expérience fut concluante, elle ne fut pas étendue, car à l'époque la nécessité de l'automatisation n'était pas aussi clairement ressentie que maintenant" ou encore "malheureusement l'intérêt d'un tel mode d'exploitation ne fut pas clairement perçu à l'époque"s.

\subsection{Le matériel pneumatique.}

En 1956, les premiers matériels pneumatiques de série équipent la ligne 11. Puis, après, ce sont les lignes 1,4 et 6 qui sont équipées de matériel pneumatique (M.P.59). Durant cette période, c'est la seule innovation majeure qui ait dépassé le stade expérimental. Avec le déblocage financier du III ${ }^{\text {ème }}$ Plan, en 1959, c'est même la généralisation du matériel pneumatique qui s'offre comme pôle principal de modernisation du réseau. Le pneu est censé résoudre "tous" les problèmes :

"Dans ce domaine, la Régie Autonome des Transports Parisiens a adopté une mesure que l'on peut, à bon droit, considérer comme

4 Développement et perspectives de l'expérience de pilotage automatique des rames du métropolitain de Paris", D. Sutton (ingénieur en chef adjoint au service des études techniques de la RATP), in Revue Générale de l'Électricité, février 1969, t. 78, p. 135.

5 Les dernières réalisations de la RATP en matière de pilotage automatique", Stablo (ingénieur en chef adjoint), Leroy (ingénieur en chef), in Revue Générale des Chemins de Fer. septembre 1972.

R. Foot (1988) Automatisation du métro et qualité de service Cahier du GDR Réseaux, 11: 36-59 
révolutionnaire, en introduisant les pneumatiques gros porteurs dans les bogies de voitures du métropolitain; l'emploi de ces pneumatiques rend pratiquement le roulement des voitures parfaitement silencieux; il est le seul à apporter le silence, non seulement à l'usager à l'intérieur des voitures, mais également sur les quais des stations, et, dans le cas des lignes aériennes, aux riverains.

Il n'est pas discutable d'autre part, que le matériel sur pneus, grâce à ses hautes performances dues à la grande adhérence du caoutchouc, permet d'augmenter à peu de frais le débit des lignes et, par voie de conséquence, de valoriser un réseau qui a été dépassé en un certain nombre de points par le développement extraordinaire du trafic" 6

Encore faut-il ajouter qu'il est prévu de "porter progressivement à 6 puis à 7 le nombre de voitures des trains, limités actuellement à 5" 7 afin d'augmenter de manière conséquente l'offre de transport. Mais l'augmentation de l'offre par augmentation de la longueur des rames entraîne des coûts considérables puisqu'il faut intervenir sur la longueur des quais. Dans l'immédiat, ce sont les qualités du pneumatique qui doivent résoudre les problèmes d'un "réseau dépassé par le trafic voyageur". L'argumentaire en faveur de la généralisation du pneumatique peut se scinder en deux :

- d'une part le confort du voyageur et des riverains ;

— d'autre part l'augmentation de l'offre par diminution des temps de parcours.

Pour que le confort du roulement sur pneumatique puisse être apprécié, il aurait fallu qu'effectivement le pneu permette une amélioration sensible de l'offre de transport, ce qui n'a pas été le cas. Depuis au moins 1930, les ingénieurs de la RATP (ex CMP), ont mis en évidence que l'augmentation du débit des rames passait principalement par le contrôle des temps d'arrêt en station. La réduction

6 La RATP au service de 8 millions de Parisiens”, BID, novembre-décembre 1962, p. 23.

7 La RATP au service de 8 millions de Parisiens", BID, novembre-décembre 1962, p. 23

R. Foot (1988) Automatisation du métro et qualité de service Cahier du GDR Réseaux, 11: 36-59 
des temps de parcours en interstation n'a qu'un effet marginal sur le temps de parcours global. Effet marginal qui d'ailleurs ne peut être effectif que s'il y a contrôle du temps de stationnement. En plus de cela, l'argument d'une augmentation du débit "à peu de frais" n'est pas réel. Le passage au pneu suppose une modification totale de la voie. Modification qui, du fait des heures d'ouverture du réseau, est complexe et délicate à réaliser ${ }^{8}$.

\section{L'HYPOTHESE SOCIALE D'UN CHOIX TECHNOLOGIQUE.}

Ce choix du pneumatique au détriment des autres innovations ne peut être expliqué en dehors de la configuration spécifique du travailleur collectif d'ensemble du réseau ferré de la RATP et, en premier lieu, de ses dirigeants et concepteurs.

En effet, le bilan des quatre expériences du point de vue de ce qu'un choix technique suppose comme reconfiguration de l'organisation du travail, met en évidence :

la moins coûteuse et peut-être la plus efficace, la centralisation de la régulation, ne connaîtra pas d'extension jusqu'en 1965. Pour améliorer la régulation, ce système implique une réorganisation du collectif de ligne qui soumette la conduite individuelle d'une rame à un point de vue plus global sur l'état du mouvement et de la circulation des rames sur une ligne ;

- le Pilotage Automatique ne connaît pas non plus d'extension jusqu'en 1965. L'expérimentation est purement et simplement arrêtée ;

- le matériel articulé est abandonné pour des raisons de rigidité due à sa conception. L'allongement des rames par adjonction d'un

8 C'est pourquoi, d'ailleurs, à partir du milieu des années soixante, l'option fer a prévalu sur le pneu.

R. Foot (1988) Automatisation du métro et qualité de service Cahier du GDR Réseaux, 11: 36-59 
élément supplémentaire ne peut se faire que par des allongements immédiatement importants des quais ;

- le matériel pneumatique qui n'offre pas d'amélioration solution réelle en ce qui concerne l'amélioration de l'offre est par contre adopté.

On constate donc que les solutions techniques supposant une réorganisation d'ensemble (centralisation des communications) au profit du Mouvement sur la Traction et/ou supprimant l'aspect conduite comme base professionnelle de la filière Traction (le Pilotage Automatique et les conducteurs) sont soit éliminées soit confinées à une seule ligne. Par contre, l'axe privilégié dans la modernisation est celui qui fait porter les améliorations sur le renforcement de l'aspect individuel de la conduite tant pour la vitesse (pneu) que pour la productivité apparente (allongement des trains). Ce constat ne signifie pas forcément que le poids, le rapport de force des conducteurs ait été prépondérant dans ce choix d'orientation. L'explication réside plutôt dans la réalité corporatiste de la Traction comme organisation sociale inter-hiérarchique des conducteurs aux ingénieurs et dirigeants. Le renforcement du rôle des conducteurs est la forme d'apparition phénoménale du poids et de l'importance des ingénieurs Traction vis-à-vis de l'ensemble des autres dirigeants. L'augmentation du rôle du conducteur devient l'expression médiatisée des capacités technologiques des ingénieurs en ce qui concerne les matériels roulants. La survalorisation de la conduite et par conséquent des conducteurs et de leur professionnalité est la traduction sociale, dans l'espace de la production, d'une sure présentation par les concepteurs du rôle du matériel roulant et de ses performances individuelles dans un système collectif de production de transport. Cette orientation technique du métro met en évidence que la régulation de l'offre de transport n'est pas, à cette époque, considérée comme centrale dans l'organisation de la production.

R. Foot (1988) Automatisation du métro et qualité de service Cahier du GDR Réseaux, 11: 36-59 


\section{L'EXPULSION DES AGENTS DE LA REPRESENTATION TECHNIQUE.}

Les orientations techniques choisies à la fin des années cinquante et au début des années soixante, laissent de fait en l'état, la structuration du travailleur collectif.

Or, les retards en ligne comme les conditions de transport des voyageurs ne s'améliorent pas. Le fit que la régulation ne s'érige pas comme élément déterminant de la qualité de service n'a pas permis que les virtualités techniques se transforment en réalité sociale.

Une remise en cause radicale des modalités de fonctionnement du réseau apparaît et s'impose entre 1964 et 1967. La dégradation, devenue inacceptable, du réseau permet à l'idéologie cybernétique de devenir la base fondatrice de la transformation du système socio-technique du métro. On passe d'une survalorisation du rôle des conducteurs à une volonté d'expulsion des agents par substitution de rapports automatiques des techniques entre elles aux rapports sociaux de travail.

Dans le cas de la RATP, conséquence apparente de l'immobilisme social et organisationnel, à la technique est dévolu le rôle de transformer le système d'ensemble. Dès le départ, la technique semble s'inscrire comme projet autonome des rapports sociaux réels. L'initialisation du projet porte dans sa matérialité ses lignes futures de réalisation et de développement. D'emblée, il s'agit d'automatiser la conduite, de programmer la circulation tant en ligne que dans la gare, la vente des billets et l'admission des voyageurs.

En 1966, avant même qu'une expérimentation "grandeur nature" n'ait été effectuée, la centralisation de tous les moyens d'action et de contrôle de la ligne sur une seule personne semble déjà dépassée :

"Il n'est pas interdit de penser que la commande centralisée que nous allons prochainement mettre en service pourrait constituer une étape appelant une automatisation ultérieure plus complète où un cerveau

R. Foot (1988) Automatisation du métro et qualité de service Cahier du GDR Réseaux, 11: 36-59 
électronique viendrait finalement prendre en charge la quasi totalité des actions encore laissées à l'initiative humaine" ${ }^{9}$.

Très rapidement, une fois l'implantation de ce nouveau système réalisée en juin 1967 sur la ligne 1, les premières déconvenues apparaissent, en particulier pour ce qui concerne la régulation automatique. Dans la même année, une recherche d'un autre type de régulation du mouvement des rames, basé sur le contrôle du temps de stationnement est mis en branle. Des éléments considérés comme secondaires se révélèrent principaux (par exemple: la Téléphonie Haute Fréquence) et inversement.

Malgré ces premiers indices d'un décalage entre la conception théorique et la réalité de l'exploitation qui ont amené à des réaménagements dans la réalisation du projet de modernisation, sa cohérence d'ensemble, à terme, apparaît encore inscrite dans une forme de fascination technicienne vis-à-vis du progrès et de ses potentialités.

Les réaménagements du projet s'inscrivent dans un projet encore plus globalisant. Cela est particulièrement visible en ce qui concerne la circulation des voyageurs :

"L'automatisation de la distribution et du contrôle des titres de transport (...) va nous conduire à équiper chaque station ou groupe de stations d'un petit calculateur qui recevra en temps réel toutes les informations sur les ventes de billets et sur le passage des voyageurs. Il est tentant de profiter des informations ainsi rassemblées pour renseigner directement le système central de gestion de l'entreprise, en temps réel ou faiblement différé, sur l'état de la comptabilité des ventes et sur le trafic des voyageurs. Les calculateurs des PCC de lignes possèdent de leur côté toutes les informations sur les mouvements des trains. Un réseau de transmission de données reliant les calculateurs de péages des stations, les calculateurs des PCC des

9 "La modernisation des méthodes de l'exploitation du Métropolitain", Notice IT -130-66, juillet 1966, p. 5

R. Foot (1988) Automatisation du métro et qualité de service Cahier du GDR Réseaux, 11: 36-59 
lignes et le système central de gestion permettra donc d'élaborer dans un temps record la comptabilité des recettes et toutes les statistiques désirées sur le trafic des voyageurs et le mouvement des trains" 10 .

Après le mouvement des trains, celui des voyageurs et de l'argent, c'est au tour du personnel d'être saisi, en projet, par le système central :

"On envisage aussi d'utiliser ce réseau pour transmettre les informations nécessaires pour la gestion du personnel: pointage des heures de service, définition des postes de travail..." 11.

De tous ces projets, il se dégage une représentation du rôle des agents par les ingénieurs qui correspond essentiellement à une fonction de surveillance des automates même si celle-ci est valorisée par le terme d'Inspecteur. Cette représentation illustre une illusion quant à la réalité des processus réels de mises en oeuvre d'un procès de production, de l'écart entre le travail théorique et la réalité des procès de travail. C'est sur la base de ce "malentendu" que J'on retrouve dans les écrits des ingénieurs, la définition de l'automatisme par Baudrillard :

"L'automatisme est ainsi comme une clôture, une redondance fonctionnelle, expulsant l'homme dans une responsabilité spectatrice" 12

$\mathrm{Au}$ milieu des années 70 , ce processus de fuite en avant technique s'interrompt. Le rêve d'une expulsion définitive des agents hors du procès de production, par la technique, s'achève dans l'épreuve des faits. Mais de cette période, où la technique est le vecteur principal de la restructuration organisationnelle des rapports de travail et de production, le non-dit, la non-verbalisation l'emporte

10 "L'automatisation du Métropolitain de Paris: buts et perspectives d'avenir", L. Guiesse (chef du service des études techniques à la RATP), in Revue Générale d'Electricité, t. $78, \mathrm{n}^{\circ} 2$, février 1969 , p. 146 et 148.

$11 \mathrm{Ibid}$, p. 147. Cette volonté centralisatrice ne correspond pas au seul souci d'ordre. Elle s'appuie principalement sur le désir de centraliser la maintenance des calculateurs.

12 Baudrillard, Le système des objets. Gallimard, 1984, p. 155.

R. Foot (1988) Automatisation du métro et qualité de service Cahier du GDR Réseaux, 11: 36-59 
sur l'explicitation des causes réelles de l'amélioration du transport, sur le dévoilement des points essentiels du processus de modernisation.

Ce phénomène débouche sur la pérennisation d'une dualité entre l'image publique du réseau métro qui se structure dans ses traits techniques et une image privée, trouble et floue, qu'aucun des acteurs ne parvient à stabiliser, à en saisir les traits essentiels.

\section{LA POLARISATION DU RESEAU ENTRE LA QUALITE DE L'ACCUEIL ET LA CIRCULATION DES RAMES.}

La conception du métro lyonnais va s'enraciner dans cette image publique, image théorique du fonctionnement d'un réseau métro, diffusée principalement par le milieu professionnel du métro. Mais il ne s'agira pas d'une simple transposition. Sur cette base théorique, le réseau lyonnais va exacerber certains aspects du modèle. Cette exacerbation du modèle trouve pour partie son origine dans l'histoire de la création de ce nouveau réseau, celui du métro.

Une nouvelle technique de transport en commun est introduite au sein d'un réseau existant dont la culture technique est profondément liée au bus et au tramway, à des systèmes de transport de surface. Avant même que le nouveau réseau existe cela pose le problème de la connaissance et de la maîtrise de ce nouveau mode de transport en commun par ceux qui en auront la charge. Un réseau métro à la différence d'un réseau de bus ne s'adapte pas à son environnement urbain hormis dans la définition de son tracés, il crée de toutes pièces son propre environnement ce qui entraîne une obligation de prédéfinition des modalités d'exploitation dès les travaux de génie civil. Ce sont ces derniers qui fixent la base du niveau de flexibilité et de performances de l'exploitation future du réseau. La configuration du souterrain pour les terminus, la ligne et les stations imposent le cadre et la base du type d'exploitation du réseau de transport.

Les dimensions du souterrain en ligne, au terminus et en stations, fixent de manière durable à la fois les spécifications du matériel roulant (largeur, hauteur,

R. Foot (1988) Automatisation du métro et qualité de service Cahier du GDR Réseaux, 11: 36-59 
longueur et modularité), les modalités d'accès au transport pour les voyageurs (parcours de la voie publique au métro, aménagement des rames) et le mode d'exploitation lié au type de régulation aux potentialités de cadencement des rames.

Cette plus grande liberté de définition se traduit par une plus grande exigence a priori dans la connaissance de l'exploitation d'un réseau métro afin de définir ses caractéristiques. Le réseau lyonnais se trouvait donc dans la situation paradoxales qu'à une plus grande maîtrise potentielle d'un système de transport correspondait un moindre savoir-faire.

Ce problème se posait avec d'autant plus d'acuité à Lyon qu'au début du projet, en 1964, le comité du Syndicat des Transports en Commun de la Région Lyonnaise

(STCRL) s'est tourné vers la filiale ingénierie de la RATP, la SOFRETU, pour mener à bien les études prospectives sur le réseau de Lyon. Mais en 1968, une société est créée, la SEMALY, pour piloter le projet métro et plus tard, l'exploiter. Cette situation où la SEMALY se posait en concurrent de la SOFRETU sur son terrain privilégié, celui du métro, n'a apparemment pas contribué ni à l'établissement de bons rapports de travail ni a fortiori il des transferts de compétences et de savoir-faire entre la SEMALY et la SOFRETU.

De plus, la modification de l'attribution de la responsabilité de l'exploitation du réseau métro en cours de travaux n'a pas contribué à une prise en charge de l'organisation sociale du travail dans le projet technique. Au début des travaux, en 1973, c'est toujours la SEMALY qui était pressentie pour la gestion future des lignes de métro. Il faut attendre 1975, date d'arrivée de la rame prototype pour que la décision de transfert de compétences, en matière de gestion, de la SEMALY vers TCL soit prise, à l'initiative du Syndicat des Transports de l'Agglomération Lyonnaise (SYTRAL).

R. Foot (1988) Automatisation du métro et qualité de service Cahier du GDR Réseaux, 11: 36-59 
Cette dernière était soucieuse de conserver une homogénéité de direction aux réseaux de transport en commun de la région lyonnaise ${ }^{13 .}$

Si cette situation a pu être résolue entre TCL et la SEMALY, en particulier par le transfert d'un responsable du projet de la SEMALY vers TCL, il semblerait que les rapports de "concurrence" entre la SEMALY et la SOFRETU aient eu des répercussions plus durables car ils auraient conduit à un blocage du transfert d'expertise dont les conséquences peuvent encore se lire dans la conception matérielle du réseau métro. Un des symptômes de ce phénomène de blocage dans les transferts d'expertise se révèle dans la conception des appareils de voie en ligne permettant en théorie la réalisation de services provisoires mais qui dans la pratique s'avère être de peu d'utilité. Un autre est constitué par la configuration des terminus à une seule voie de garage où seule la manoeuvre trottoir peut être effectuée. Cette dernière option a été prise pour éviter des surcoûts $^{14}$ importants en matière de génie civil qu'aurait entraîné une configuration de terminus à trois voies; elle implique une limitation de la fréquence des rames en ligne qu'aucun système technique ne peut surpasser et entraîne de graves perturbations en cas d'avarie de matériel roulant sur la voie unique.

Les options prises en matière de génie civil et de tracé des voies nous indique déjà que dans sa conception le métro lyonnais a sous-estimé l'importance, dans le fonctionnement d'une ligne de métro, de certains points névralgiques, ceux qui déterminent les potentialités de cadencement des rames en ligne. Cela s'est traduit par une surestimation des capacités de circulation des rames qui a provoqué la commande de matériel roulant excédentaire. Les tentatives de diminuer l'intervalle minimum entre deux rames sont venues en butée sur la

$13 \mathrm{Au}$ moment de l'apparition d'une nouvelle technique de transport en commun la question de l'unicité de direction de l'ensemble des réseaux n'est pas une simple question d'école. A Lille, le VAL s'est constitué en réseau autonome. Les effets pervers de cette situation ont fini par émerger et la décision d'unifier dans une même structure organisationnelle les réseaux hétérogènes du point de vue technique a été prise.

14 Dans la construction d'un métro, on évalue en général la répartition des coûts entre le génie civil et les équipements (installations fixes, matériel roulant...), à un rapport de 80/20 de l'ensemble du coût.

R. Foot (1988) Automatisation du métro et qualité de service Cahier du GDR Réseaux, 11: 36-59 
configuration des terminus. En 1983, sur les 32 rames disponibles dont 11 proviennent d'une commande effectuée un an après la mise en service du métro, en 1979, 21 sont en service en heure de pointe sur les lignes A et B, 2 sont en réserve opérationnelle et 9 sont en excédent.

Par contre, l'aménagement des stations, la conception du péage ouvert et le confort des rames sont ressentis comme une réussite par les voyageurs. La circulation des voyageurs dans les accès et dans les rames est considérée comme agréable et facile.

Tout se passe comme si la conception du métro avait pu intégrer dans le génie civil la partie d'ouverture du réseau vers le public sans avoir su optimiser la partie de prise en charge des usagers dans les rames en mouvement, comme s'il avait été décidé de privilégier la circulation autonome des voyageurs sur la circulation des voyageurs prise en charge par la société exploitante. Le symptôme perceptible de ce phénomène peut être observé dans le refus d'avoir une surlargeur en terminus permettant d'avoir trois voies, condition nécessaire à un abaissement de l'intervalle, tandis que sur toute la longueur des lignes, le choix s'est porté sur une largeur de tunnel permettant la circulation de rames d'une largeur supérieure d'environ $50 \mathrm{~cm}$ à celle de Paris $(2,90 \mathrm{~m}$ contre $2,43 \mathrm{~m})$.

Cette opposition, qui pour l'heure n'a pas de conséquences sensibles sur la qualité du service offert, révèle que le processus permettant une réelle maîtrise de la conception d'un réseau métro suppose une accumulation d'expérience, une continuité dans la mise en oeuvre des processus d'innovation et que le système de régulation et sa logique ne sont pas des éléments naturels, des données mais bien une production sociale.

Sur un autre point, celui de l'intégration des conducteurs dans la production de transport, on va voir apparaître des processus contradictoires entre la finalité de la production et la définition du procès de travail des conducteurs telle qu'elle s'inscrit dans la technique ou la théorie.

R. Foot (1988) Automatisation du métro et qualité de service Cahier du GDR Réseaux, 11: 36-59 


\section{LES CONDUCTEURS INFORMULES ET LE PILOTAGE AUTOMATIQUE.}

L'option prise, en matière sociale par le réseau de transport en commun de Lyon, a été de conserver une parité de salaire et de classification entre les conducteurs de surface et du métro afin de permettre une circulation, une mobilité des agents d'exécution et de maîtrise entre les deux réseaux. Pour atteindre ce résultat et compte tenu de l'image et des effets apparents de la division salariale à la RATP, le réseau lyonnais opte pour une définition restrictive de la fonction de conducteur en ce qui concernent le traitement des avaries en ligne. Ce sera aux régulateurs du PCC qu'il incombera de définir et de prescrire aux conducteurs les interventions qu'ils auront à effectuer pour reconfigurer une rame. Le conducteur est ainsi défini comme surveillant d'automate, émetteur d'informations et exécuteur de prescriptions. Cette définition de la fonction de conducteur est émise afin d'éviter l'émergence de revendication prenant appui sur une différence dans le travail théorique prescrit entre les conducteurs de surface et du métro. Il s'agit d'une forme d'éradication préventive de toute capacité à se prévaloir d'un attribut technique réel pour légitimer une forme salariale de particularisme, de catégorialisme. Le problème est que, dans ce domaine du traitement d'avarie, la configuration technique du procès de production fait du conducteur l'intervenant quasi "naturel". Il y a donc là, une opposition entre la définition technique et la définition salariale du conducteur de métro. Cette opposition va se révéler d'autant plus forte que la qualité des transmissions par téléphonie haute fréquence entre les rames et le PCC est très mauvaise et oblige les régulateurs et les conducteurs à se faire répéter les communications. Il va sans dire que cette mesure ne va pas dans le sens d'une optimisation de la circulation des rames !

Cette mesure, pénalisante en terme de qualité de service dans les états dégradés du réseau et pour cette raison de nombreuses fois contournées par les régulateurs et les conducteurs, ne semble pas réellement efficace dans sa logique salariale. En effet, les conflits qui ont éclatés sur la question d'une différenciation salariale du groupe des conducteurs de métro vis-à-vis des autres conducteurs, en particulier celui de 1979, ont été résolus sans déroger au principe de la parité

R. Foot (1988) Automatisation du métro et qualité de service Cahier du GDR Réseaux, 11: 36-59 
salariale car il existait aussi, de la part des syndicats majoritaires (FO et la CGT), une volonté de conserver une unité salariale entre les différents réseaux.

Par contre dans la définition technique du système de contrôle de la circulation des rames, il apparaît une opposition entre principe et technique de production due au manque de fiabilité du Pilotage Automatique (PA). Ce n'est pas le système de $\mathrm{PA}$ en fonctionnement à Paris qui est retenu, du fait des modalités de passage d'un marché global avec le groupement d'entreprise lauréat du concours.

Pour ce groupement, la mise au point du PA correspond à une première et les difficultés de réalisation son sous-estimées. Dans la perspective d'un fonctionnement satisfaisant du PA, aucune signalisation d'espacement en ligne, intégrée au système de sécurité et visible par le conducteur n'a été prévu. C'est à la demande des exploitants, qu'au dernier moment, avant l'ouverture du métro, une signalisation auxiliaire est mise en place. Celle-ci ne correspond pas à une signalisation de type ferroviaire, en sécurité intrinsèque, mais à une signalisation d'espacement indiquant, en tête de quai, l'occupation ou la libération de la voie dans la station suivante. Elle ne permet donc ni une optimisation des intervalles ni un fonctionnement sécuritaire, matérialisée dans le système technique.

Heureusement que cette modification de dernière minute a été effectuée car il faudra attendre 1985 pour que la fiabilisation du PA soit obtenue. Durant cette période et surtout entre 1978 et 1983, les conducteurs rouleront la plupart du temps en Conduite Manuelle sur Signalisation Auxiliaire sans que l'on puisse dire que la conduite manuelle a, en soi, perturbée la régularité des rames. D'une certaine manière, on peut dire que la défaillance technique du PA permet de s'interroger sur la nécessité de celui-ci pour assurer une bonne qualité de service. Mais cette question n'a jamais été réellement formulée.

Sur ce point, le réseau de la RATP a été confronté au même problème. La nécessité du Pilotage Automatique pour toutes les heures de la journée fut remise en cause tant pour répondre à une demande syndicale que pour tenter de remédier à la montée de la désaffection au travail.

Au début, elle consiste en la reprise d'un projet de 1966 de mise en concurrence du Pilotage Automatique avec la conduite manuelle qui aboutit à une

R. Foot (1988) Automatisation du métro et qualité de service Cahier du GDR Réseaux, 11: 36-59 
expérimentation sur la ligne 6 . Pour des raisons d'opposition à cette mise en concurrence des automates et des hommes mais surtout pour des raisons techniques ${ }^{15}$ concernant la qualité de service dans les états dégradés du réseau, ce projet ne fut pas maintenu.

Finalement, le projet de Conduite Manuelle Contrôlée retenu en 1978 et généralisé à partir des années 80 ne retiendra que deux fonctions:

- contrôle de vigilance du type homme mort (VACMA);

- arrêt automatique en cas de franchissement de signal fermé.

Par contre, le contrôle des vitesses s'effectue a posteriori à partir des bandes du chronotachygraphe. La Conduite Manuelle Contrôlée concrétise une stabilisation du repartage des fonctions entre le conducteur et les automatismes à l'intérieur du système d'ensemble. Mais déjà, certains modes d'usage de la Conduite Manuelle Contrôlée (en particulier en heure de pointe) remettent en cause, au moins partiellement, la corrélation apparente entre Pilotage Automatique et améliorations des conditions de transport.

Des sondages effectués à partir des bandes de chronotachygraphies et des interviews effectués montrent une tendance des conducteurs à inverser l'utilisation de la Conduite Manuelle Contrôlée par rapport à la réglementation. Théoriquement, en heure de pointe quand l'intervalle est inférieur à deux minutes, la conduite en Pilotage Automatique est obligatoire, pourtant de nombreux conducteurs utilisent la Conduite Manuelle Contrôlée sans conséquence du point du vue de la régulation ${ }^{16}$.

D'une certaine manière, cette "désautomatisation" du procès de travail confirme que la rationalité du Pilotage Automatique pour améliorer le transport est loin d'être évidente comme le montrait déjà, en 1969, l'expérience de la ligne 7 qui

15 Non fiabilité d'un système basé sur le Pilotage Automatique en cas d'avarie du Pilotage Automatique, une des causes importantes de la reprise en conduite manuelle.

16 Cf. enquête de F. De Peslouan sur "Normes de production et usages du transport", 1987, RATP.

R. Foot (1988) Automatisation du métro et qualité de service Cahier du GDR Réseaux, 11: 36-59 
sans PA, avait permis une diminution des intervalles permettant une augmentation d'environ

$15 \%$ de l'offre. Ce constat se conjuguant à la nécessité de renouveler certaines installations de Pilotage Automatique, ont même suscité la question de savoir si la suppression du Pilotage Automatique ne pouvait être envisagée sans que ni du point de vue économique ni du point de vue du public cela ne soit préjudiciable. Mais ce constat n'est pas officiellement formulé.

L'aspect paradoxal du choix du réseau lyonnais d'aller vers une automatisation intégrale de la circulation des rames provient de ce qu'il se produise au moment où dans les deux réseaux la question d'une désautomatisation partielle du procès de production aurait pu être posée de façon pertinente. C'est ce moment même qui est choisi pour ne pas formuler la question, pour projeter dans MAGGALY sa disparition, son informulation technique.

\section{L'EFFET VITRINE ET LA DESIGNATION DU CONDUCTEUR COMME UNE CONTRAINTE.}

La question de la définition du travail des conducteurs est une véritable question posée aux gestionnaires de réseaux. Il est clair que la réponse qui jusque là a été donnée n'est pas satisfaisante. La solution choisie dans la plupart des réseaux de maintenir un conducteur qui a principalement en fonction de "presse-boutons" est à l'évidence génératrice d'un malaise: à la déqualification du conducteur se conjugue le maintien d'un rapport de force virtuellement important compte tenu de la productivité du transport en commun par métro.

Mais la réponse apportée par le projet MAGGALY, les raisons qui l'ont fait adoptée ne sont pas complètement satisfaisantes. Le projet tire sa légitimité d'une double source liée par la notion de service public :

- la gestion économique du réseau ;

— la qualité du transport public.

R. Foot (1988) Automatisation du métro et qualité de service Cahier du GDR Réseaux, 11: 36-59 
Sur le premier aspect, il y a et ce n'est pas propre au seul réseau de Lyon, un malaise à définir le coût d'un réseau de transport en commun. En général, il y a une division, dans la prise en compte du fonctionnement d'un réseau, entre les investissements et les coûts de fonctionnement, d'exploitation. A s'en tenir au seul critère de gestion interne de la société exploitante qui est à la base souvent du rapport économique qu'elle entretien avec ses tutelles et pour cette raison défini avec elles, les sociétés de transport en commun se définissent principalement comme des sociétés de main d'oeuvre. Mais si cette vision donnée d'un réseau par ses normes de gestion peut être inacceptable dans le cas d'un réseau en site propre et a fortiori souterrain. Dans ce cas, en effet, ce sont, au contraire, des industries à forte concentration capitalistique et ce sont les annuités de remboursement des emprunts qu'il faut mettre en balance avec les coûts d'exploitation. Une telle mise en regard des trois termes principaux (génie civil, équipement et modalités d'exploitation) qui constituent un réseau, aurait en l'occurrence invalidé de manière définitive le projet.

Il s'est produit au contraire, sur la base d'un syllogisme, une évidence de représentation des conducteurs comme un simple coût dont la suppression permet naturellement une économie. La production de cette imagé du salarié comme simple coût s'obtient à partir de deux propositions simples: on sépare les investissements des coûts d'exploitation et on ne retient que les coûts d'exploitation. De là se déduit assez logiquement, puisque les investissements matériels ont disparu de la scène et que les frais matériels d'exploitation sont eux considérés comme incontournables, que les seuls espaces de gain dans la gestion ne peuvent être obtenus que dans un mouvement devenu irrationnel de suppression de salariés quitte pour ce faire à augmenter l'investissement technique.

Même et peut-être surtout si cette expérimentation se situe sur un segment de marché porteur d'un développement économique potentiel, il paraît contreperformant qu'une telle confusion perdure.

Sur la base de mirages, il peut se révéler que les marchés escomptés s'avèrent être des leurres surtout compte tenu des problèmes de financement que les réseaux de transport urbain rencontrent, au niveau international.

R. Foot (1988) Automatisation du métro et qualité de service Cahier du GDR Réseaux, 11: 36-59 
Sur le second aspect, celui de la qualité de service, il nous semble également que la question soit mal posée. La norme horaire des conducteurs est théoriquement calculée en fonction des périodes creuses et d'affluence sur chaque ligne. Quand il advient un état dégradé dans le fonctionnement d'une ligne, la régulation tend à diminuer la dégradation du service. Les normes horaires des conducteurs sont souvent présentées comme une contrainte empêchant la régulation optimum de la ligne dans ses états là car freinant les possibilités de glissement des horaires des rames. Ce type de raisonnement passe sous silence que si les calculs de charge et les horaires des conducteurs sont corrects, un déplacement, dans le temps d'une journée, du passage des rames, est une dégradation du service. Un retard pris en début d'heure de pointe ne se rattrape pas en fin d'heure de pointe pour les voyageurs. La contrainte que fait peser les conditions horaires de travail des conducteurs sur le fonctionnement traduit également, sous la forme d'un rapport entre agents, une obligation de ponctualité ou à tout le moins une obligation de fréquence, de capacité d'offre de transport à des heures déterminées de la journée.

La présentation d'une automatisation intégrale de la conduite comme moyen de lever la contrainte des conducteurs en matière d'horaire peut s'il n'y a pas au préalable de tentative d'objectivation du problème, tout aussi bien signifier qu'il s'agit de lever la contrainte des horaires différenciées de la demande, de supprimer le voyageur réel dans la représentation technique du transport, dans la logique de fonctionnement du système. En l'état actuel de la technique, il n'y a pas lieu d'espérer de meilleurs performances de la circulation des rames en automatique ou en manuel et les problèmes qui ne sont pas posés ou envisagés n'ont pas plus de chance d'être résolus qu'antérieurement.

A sa manière, le projet MAGGALY renoue avec la tentation technicienne, la fétichisation de la technique que la RATP a connu dans les années 60 :

“L'alternative qui s'offrirait à l'avenir serait de réaliser l'automatisation intégrale ou de revenir en arrière en limitant le degré d'automatisation et en confiant au personnel plus de responsabilité en matière de sécurité. Cette deuxième proposition me 
semble tout à fait aller à contre courant de l'histoire des transports guidés en site propre" ${ }^{17}$.

C'est dans ce sens donné à l'histoire que s'enclenche la spirale vicieuse, la dynamique d'un recours accru à la technique envisagée comme le deus ex machina qui amènerait la paix sociale par expulsion d'un des acteurs. Mais ce rêve qui fut mécanique dans les années 30 puis cybernétique dans les années 60 , qui est informatique aujourd'hui, chaque fois s'est brisé en se matérialisant dans un procès de production quotidien. Cela déplace les rôles, fait apparaître de nouveaux acteurs que jusque là les autres occultaient mais en fin de compte les mêmes problèmes restent posés. Le projet MAGGALY peut être encore l'objet d'un pari, celui de trouver une synergie réelle entre les collectifs de travail et la technique, entre l'acquisition de compétences et l'innovation, entre le présent et l'avenir et non pas simplement l'enjeu d'une fascination technicienne où, à part quelques rares élus, les acteurs de la production sont invités à un rôle de figurants dans l'histoire, dans leur propre histoire quotidienne.

17 Perspectives d'automatisation du métro de Lyon, J. Pernot (chef du service Métro de la Société lyonnaise de Transport en Commun) TCL.

R. Foot (1988) Automatisation du métro et qualité de service Cahier du GDR Réseaux, 11: 36-59 